\title{
Efficacy and safety of dapagliflozin by glycemic status in the DAPA-CKD trial
}

Frederik Persson ${ }^{1}$, Peter Rossing ${ }^{1,2}$, Priya Vart ${ }^{3}$, Glenn M. Chertow ${ }^{4}$, Fan Fan Hou ${ }^{5}$, John J.V. McMurray ${ }^{6}$, Ricardo Correa-Rotter ${ }^{7}$, Harpreet S. Bajaj ${ }^{8}$, Bergur V. Stefansson ${ }^{9}$, Roberto D. Toto $^{10}$, Anna Maria Langkilde ${ }^{9}$, David C. Wheeler ${ }^{11,12}$, Hiddo J.L. Heerspink ${ }^{3,12}$, for the DAPA-CKD study group

1. Steno Diabetes Center Copenhagen, Gentofte, Denmark

2. Department of Clinical Medicine, University of Copenhagen, Copenhagen, Denmark

3. Department of Clinical Pharmacy and Pharmacology, University of Groningen, University Medical Center Groningen, Groningen, Netherlands

4. Departments of Medicine and Epidemiology and Population Health, Stanford University School of Medicine, Stanford, CA, USA

5. Division of Nephrology, Nanfang Hospital, Southern Medical University, National Clinical Research Center for Kidney Disease, Guangzhou, China

6. Institute of Cardiovascular and Medical Sciences, University of Glasgow, Glasgow, UK

7. National Medical Science and Nutrition Institute Salvador Zubirán, Mexico City, Mexico

8. LMC Diabetes and Endocrinology, Brampton, Ontario, Canada

9. Late-stage Development, Cardiovascular, Renal and Metabolism,

Biopharmaceuticals Research and Development, AstraZeneca, Gothenburg, Sweden

10. Department of Internal Medicine, UT Southwestern Medical Center, Dallas, TX, USA

11. Department of Renal Medicine, University College London, London, UK

12. The George Institute for Global Health, Sydney, Australia

Presenting author: Frederik Persson (frederik.persson@regionh.dk)

Character count: $1,772(1,800)$

Abstracts are limited to 1,800 characters not including spaces. The character count includes the abstract title, abstract body, and any images.

Abstracts can have a maximum of two images. Each image counts as 500 characters. Tables must be uploaded as image files.

There is robust evidence that sodium-glucose cotransporter 2 (SGLT2) inhibitors reduce the risk of adverse cardiovascular and kidney outcomes. The DAPA-CKD trial (NCT03036150) demonstrated a significant risk reduction in participants with chronic kidney disease (CKD), with and without type 2 diabetes, treated with dapagliflozin $10 \mathrm{mg}$ once daily compared with placebo, as an adjunct to standard care. In this prespecified analysis, we compared the 
efficacy and safety of dapagliflozin according to baseline glycemic status. The trial included individuals with CKD with an estimated glomerular filtration rate (eGFR) of 25 to 75 $\mathrm{mL} / \mathrm{min} / 1.73 \mathrm{~m}^{2}$ and a urinary albumin-to-creatinine ratio of 200 to $5000 \mathrm{mg} / \mathrm{g}$. The primary outcome was a composite of sustained decline in eGFR of at least $50 \%$, end-stage kidney disease, or death from renal or cardiovascular causes. We analyzed results by baseline glycemic status based on the American Diabetes Association criteria for $\mathrm{HbA}_{1 \mathrm{c}}$ level $<5.7 \%$ (normoglycemia) vs. $\geq 5.7$ to $<6.5 \%$ (pre-diabetes) vs. $\geq 6.5 \%$ or a history of type 2 diabetes. Of the 4304 participants in the trial, 738 had normoglycemia at baseline; 660, pre-diabetes; and 2906, type 2 diabetes. The relative risk reduction for the primary composite outcome with dapagliflozin (hazard ratio [HR], $0.61 ; 95 \% \mathrm{Cl}, 0.51-0.72$ ) was consistent in participants with normoglycemia $(\mathrm{HR}, 0.62 ; 95 \% \mathrm{Cl}, 0.39-1.01)$, pre-diabetes $(\mathrm{HR}, 0.37 ; 95 \% \mathrm{Cl}$, $0.21-0.66)$ and type 2 diabetes (HR, 0.64; 95\% Cl, 0.52-0.79; p-interaction =0.19). Similarly, we found no evidence of effect modification by glycemic status on secondary or exploratory outcomes. The safety profile of dapagliflozin was similar across glycemic groups, with no events of major hypoglycemia or ketoacidosis in participants with normoglycemia or pre-diabetes, and no ketoacidosis in any participant treated with dapagliflozin. In conclusion, dapagliflozin reduced the risk of kidney and cardiovascular events independent of baseline glycemic status. 